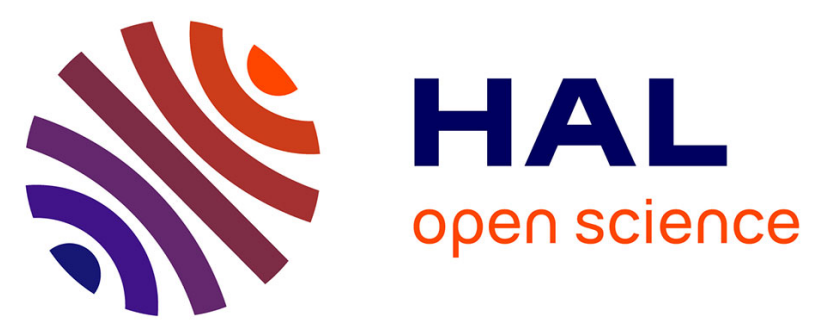

\title{
A novel force sensing platform using passive magnetic springs for mechanical characterisation of human oocytes
}

Racha Gana, Joël Abadie, Emmanuel Piat, Christophe Roux, Clotilde Amiot, Christian Pieralli, Bruno Wacogne

\section{- To cite this version:}

Racha Gana, Joël Abadie, Emmanuel Piat, Christophe Roux, Clotilde Amiot, et al.. A novel force sensing platform using passive magnetic springs for mechanical characterisation of human oocytes. Sensors and Actuators A: Physical , 2017, 262, pp.114 - 122. hal-02131247

\section{HAL Id: hal-02131247 \\ https://hal.science/hal-02131247}

Submitted on 16 May 2019

HAL is a multi-disciplinary open access archive for the deposit and dissemination of scientific research documents, whether they are published or not. The documents may come from teaching and research institutions in France or abroad, or from public or private research centers.
L'archive ouverte pluridisciplinaire HAL, est destinée au dépôt et à la diffusion de documents scientifiques de niveau recherche, publiés ou non, émanant des établissements d'enseignement et de recherche français ou étrangers, des laboratoires publics ou privés. 


\title{
A novel force sensing platform using passive magnetic springs for mechanical characterisation of human oocytes
}

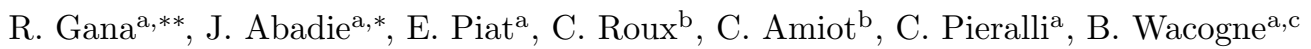 \\ ${ }^{a}$ Université Bourgogne-Franche Comté, femto-st Institute, CNRS, - ENSMM, Besançon, France \\ ${ }^{b}$ Service de Biologie et Médecine de la Reproduction-Cryobiologie, INSERM CIC 1431, \& UMR 1098 CHRU Jean Minjoz, \\ 25030 Besançon cedex France. \\ ${ }^{c}$ Centre d'Investigation Clinique de Besançon, INSERM CIC1431, CHRU de Besançon, Place Saint Jacques, 25030 Besançon \\ cedex France.
}

\begin{abstract}
This article presents a novel low cost force sensing platform for the mechanical characterisation of human oocytes. This platform is compatible with the specific context of Assisted Reproductive Technology (ART). The oocyte is placed inside a standard Petri dish filled with a culture medium. It is immobilized using a holding pipette. The disposable parts used to mechanically test each oocyte are made of glass. This configuration is very similar to the one used in IntraCytoplasmic Sperm Injection protocol excepted that the injection pipette is replaced by a glass indenter. This platform uses two passive and linear magnetic springs to measure the nanoforce applied to the oocyte. The stabilisation of the unstable magnetic springs is passively achieved using the reaction force generated by the compressed oocyte. Some preliminary results obtained with an experimental platform are presented. The global magnetic stiffness of the indenter, evaluated by simulation and experimentally identified, is about $0.0013 \mathrm{~N} \cdot \mathrm{m}^{-1}$.
\end{abstract}

Keywords: nanoforce sensor, passive magnetic springs, human oocyte characterisation

\section{Introduction}

Assisted Reproductive Technology (ART) concerns methods and techniques which help overcoming some difficulties to conceive without necessarily treating the infertility causes. Among them, in vitro Fertilization (IVF) and IntraCytoplasmic Sperm Injection (ICSI) are the most used techniques. IVF consists in putting together the oocytecumulus complex-OCC extracted from the women ovaries and the sperm inside a Petri dish and letting the fertilization happen autonomously [1]. On the contrary, ICSI forces the fertilization by directly injecting a spermatozoa inside the oocyte by means of an injection pipette [2]. The most important factor for a successful IVF or ICSI, besides the spermatozoa quality, is the oocyte quality and maturity. Indeed the oocyte selection influences the embryo development and therefore has an impact on the ART accomplishment [3].

Usually, the oocyte candidate for fertilization is determined by means of visual estimation. The clinician bases his selection on morphological characteristics such as the

\footnotetext{
* Principal corresponding author.

** Corresponding author.

Email addresses: racha.gana@femto-st.fr (R. Gana), joel.abadie@femto-st.fr (J. Abadie), emmanuel.piat@ens2m.fr (E. Piat), chistian.pieralli@univ-fcomte.fr (C. Pieralli), bruno.wacogne@univ-fcomte.fr (B. Wacogne)
}

shape, the size, the surface of the oocyte and the integrity of the first polar body [4]. However, the results obtained with this subjective and inaccurate approach [5] are quite minor compared to the arduousness of the hormonal treatment proposed to women and to the cost of the intervention. To overcome this problem, different researches were conducted in order either to improve the above mentioned morphological approach or to develop new characterization methods. Among the methods aiming at improving the morphological approach, one can mention the automatic determination of the oocyte quality using image processing $[6,7]$ and polarized light (PolScope) [8]. Among the new approaches which are not based on the morphological characterization, optical spectroscopy seems promising to assess the oocyte quality: transmission spectra of oocytes are used as a criterion to qualify their maturation stage $[9,10]$. Another promising approach concerns mechanical characterizations. These methods establish a connection between the stiffness of a living cell and its biological properties. A recent study has demonstrated that the mouse embryo's mechanical stiffness is a decisive criterion for zygote quality determination [11]. Yenez and al. proved that there is a difference between the viscoelastic properties of viable and non-viable zygotes. In the same context, several studies adopted the mechanical approach in order to prove that the stiffness should reveal some oocyte physical properties and could then be used as a possible parameter for the oocyte selection. Because oocytes exhibit very 
low stiffness, experimental implementation requires efficient tools like nanoforce sensors to achieve very low measurement range. Many devices were developed for this purpose. For example, Wacogne et al. and Liu et al. proposed a system consisting in compressing the oocyte against one or several flexible beams using a holding pipette $[12,13]$. Other researchers proposed the study of the mechanical deformation using the injection pipette used in ICSI [14, 15]. Systems based on magnetic force sensors were also developed. One can mention the robochip system [16] which consists in compressing the oocyte against a magnetic force sensor using a probe, or the micro and nanoforce sensing platform based on passive magnetic springs [17, 18] which compresses the oocyte against the border of a well located at the center of a specifically designed Petri dish. These studies have demonstrated that the mechanical behavior of an oocyte evolves and depends on its maturity stage [12, 13, 19, 17]. However, and at least in France, using these devices with human oocytes requires the approval of the "'Agence de Biomédecine"' (public organization under the supervision of the french Minister of Health) which may be difficult. Indeed, the French law No2011-814 concerning bioethics imposes strict and conservative regulations on the use of new tools in the context of ART. All components in contact with the human oocyte must imperatively be disposable and non-gametotoxic in order to avoid contamination. The oocytes must be individually manipulated in a sterile environment and the temperature of the culture medium must be controlled. In practice, only disposable Petri dishes approved for IVF procedure and glass-made tools are generally authorized. Finally, on a financial point of view, the cost of both the characterization device and the disposable parts is also an important issue. Only low cost disposable parts will be accepted by ART laboratories. As an example, the price of a disposable holding pipette is $20 €$. This price gives an idea of what should be the final cost of the disposable parts in a characterization device based on nanoforce measurement. To satisfy these challenging requirements, the development of a new low cost device is needed.

This paper presents a new platform for human oocyte mechanical characterization using a nanoforce sensor based on passive magnetic springs. A passive sensor was chosen in order to reduce the complexity of the device, while magnetic springs were chosen because of their insignificant cost, high linearity and low stiffness. All passive magnetic springs exhibit one unstable direction which is always passively stabilized using an additive repulsion force field which is necessarily based on a non-magnetic principle (in order to respect the Earnshaw's theorem). Several principles were used in the past to generate this repulsion force field like the diamagnetism (generated by diamagnetic plates [20]) or the up-thrust buoyancy (generated by a liquid [18]). To simultaneously simplify the design and respect the bioethics requirement, the originality of the design proposed in this article is that no repulsion force field

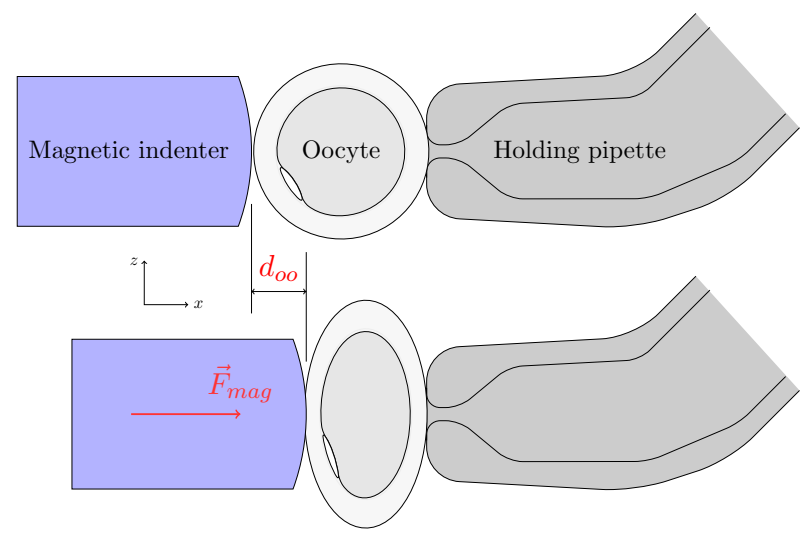

Figure 1: Principle of the mechanical characterisation of the oocyte

is required to stabilize the sensor when no measurement is performed. Furthermore, the unstable direction of the springs is chosen collinear to the measurement direction. In measurement conditions, the unstable direction is passively stabilized by the oocyte reaction force. Indeed, this is the characterized object itself which stabilizes the sensor. Such approach to stabilize passive magnetic springs is new for nanoforce sensors based on this technology.

The experimental platform as well as the oocyte mechanical characterization process is described in section 2 . In section 3, the magnetic modelling of the nanoforce sensor is developed. Some results concerning the simulation of the magnetic behavior of the indenter (the sensor's transducer) are presented. Preliminary experimental results of mechanical characterizations done with a human oocyte are presented in section 4. Finally, a calibration approach of the nano-force sensor is detailed in section 5 .

\section{Description of the platform}

\subsection{Mechanical characterisation of oocytes}

The mechanical characterization consists in determining the oocyte mechanical response during a compression cycle by using a magnetic indenter which applies a controlled compression force $F_{\text {mag }}$ on it. Figure 1 shows the configuration of the oocyte inside the platform. This configuration is very similar to the one used in ICSI procedure, except that the injection pipette is replaced by a glass indenter.

The oocyte to be characterized is placed inside a culture dish filled with a culture medium ${ }^{1}$ for providing isolation from the environment. The oocyte is immobilized using a holding pipette to prevent spurious movements during the measurement. It is compressed by a magnetic indenter placed at the opposite side of the holding pipette (see figure 1 ). The magnetic force $\vec{F}_{\text {mag }}$ applied on

\footnotetext{
${ }^{1}$ Vitrolife: medium for in vitro fertilization, culture and transfer of embryos.
} 


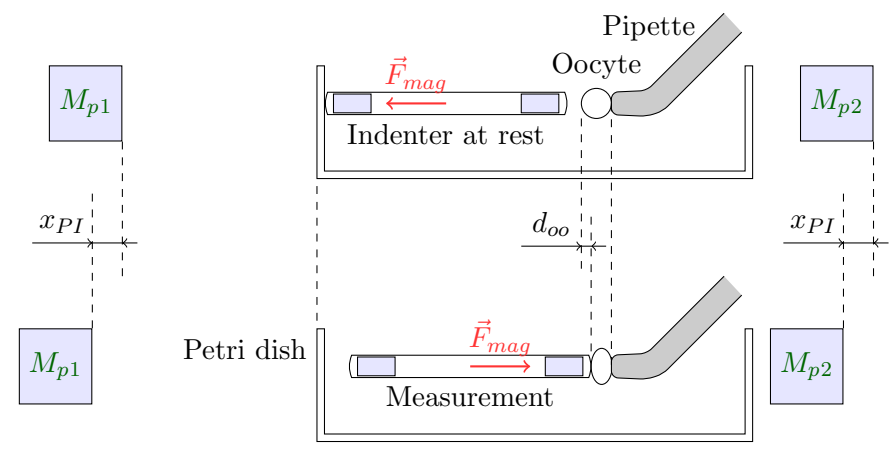

Figure 2: Operating principle of the loading device.

the oocyte is controlled by two mobile magnets $M_{p 1}$ and $M_{p 2}$ placed on Physik Instrumente (PI) micro translation stages (see figure 2). These two magnets are placed near but outside the culture dish ${ }^{2}$. The measurement of the magnetic force $\vec{F}_{\text {mag }}$ generated by the loading device and the measurement of the compression length $d_{o o}$ defined in figure 1 provide the oocyte mechanical response during the compression cycle.

\subsection{Operating principle of the force device}

The loading device uses a $6-$ DOF magnetic indenter which comes under the influence of the magnetic field generated by the two mobile magnets $M_{p 1}$ and $M_{p 2}$ (see figure 2). This magnetic influence acts as mechanical springs connected to the indenter. The configuration proposed here is similar to an indenter connected to two orthogonal springs oriented in $y$ and $z$ directions. They keep the indenter aligned along the $x$ axis. Nevertheless, in this $x$ direction which is the measurement axis, the magnetic spring has the particularity to have a negative stiffness. Therefore, the indenter exhibits an unstable behavior along $x$ and moves until it come in contact with either the Petri dish border (if it moves to the left) or the oocyte (if it moves to the right) (see figure 2). The direction of this instability along the $x$ axis can be chosen by an appropriate displacement of the magnets $M_{p 1}$ and $M_{p 2}$. When no oocyte is present, the indenter stays in contact with the Petri dish border. The instability direction is reversed when an oocyte is placed against the indenter and when magnets $M_{p 1}$ and $M_{p 2}$ are translated. Therefore, the indenter compresses the oocyte which generates a repulsive force. The latter is used to stabilize the passive magnetic spring. Consequently, an "external" repulsive force field (usually generated by diamagnetic plates located very close to the magnetic indenter like in [20]) is not necessary with this approach. This innovative and minimalistic solution has been adopted because the use of diamagnetic plates inside the culture medium presents an important risk of gametotoxicity. The magnetic indenter is composed of a glass capillary which contains two magnets with a diameter of $500 \mu \mathrm{m}$. These magnets are placed and fixed

\footnotetext{
${ }^{2}$ BD Falcon Petri Dish 50x9 - ref 351006
}

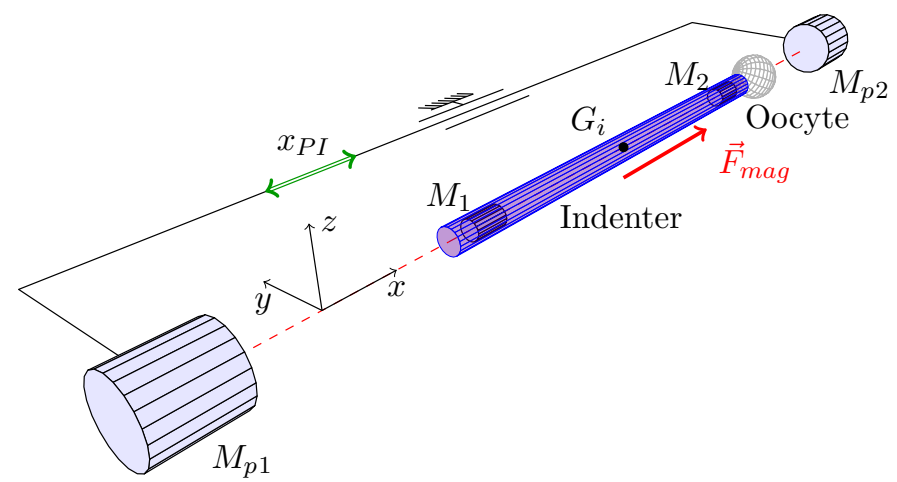

Figure 3: Principle of the magnetic indenter control using a translation stage. The holding pipette is not represented on the right of the oocyte.

horizontally inside the capillary near each extremity using glue. Finally, the capillary extremities are made gametocompatible by glass coating. The indenter is placed along the $x$ axis between the magnet couple $\left\{M_{p 1}, M_{p 2}\right\}$ which can be placed at any desired position $x_{P I}$ (see figure 3 ) using a translation stage. The distance $d$ between $M_{p 1}$ and $M_{p 2}$ is kept constant. The role of an $x_{P I}$ adjustment is to control the force $\vec{F}_{\text {mag }}$ applied on the oocyte. The magnetic influence of the external magnets $M_{p 1}$ and $M_{p 2}$ over the internal magnets $M_{1}$ and $M_{2}$ is modelled and characterized in section 3 .

\subsection{Experimental platform}

The experimental platform shown in figure 4 was specifically developed to perform a low cost mechanical characterization of human oocytes. It is composed of a Petri dish filled with a culture medium controlled in temperature with a thermoplate. The oocyte and the indenter are placed in the Petri dish. A holding pipette controlled by an Eppendorf manipulator is used to immobilize and manipulate the oocyte. A vision system is placed above the Petri dish to provide a view of the oocyte during its characterization. It is composed of a camera (Guppy F-046B), a long focal microscope objective $(\times 5, \mathrm{f}=200 \mathrm{~mm})$ and a tube provided with a zoom. The main characteristics of the loading device components are:

- magnetic indenter: $0.85 \mathrm{~mm}$ diameter and $2 \mathrm{~cm}$ length glass capillary containing two cylindrical magnets $M_{1}, M_{2}$ of $500 \mu \mathrm{m}$ diameter and $1 \mathrm{~mm}$ length,

- displacement device: two cylindrical magnets $M_{p 1}$, $M_{p 2}$ of $10 \mathrm{~mm}$ diameter and $10 \mathrm{~mm}$ length, each one located on motorized and synchronized singleaxis micro-positioning stages (Physik Instrumente M122). The stages provide a correct speed control for the displacement of $M_{p 1}$ and $M_{p 2}$. The distance $d$ described in figure 5 is adjusted once, in order to provide a correct stiffness to the magnetic spring which generates $F_{\text {mag }}$. 
(a)
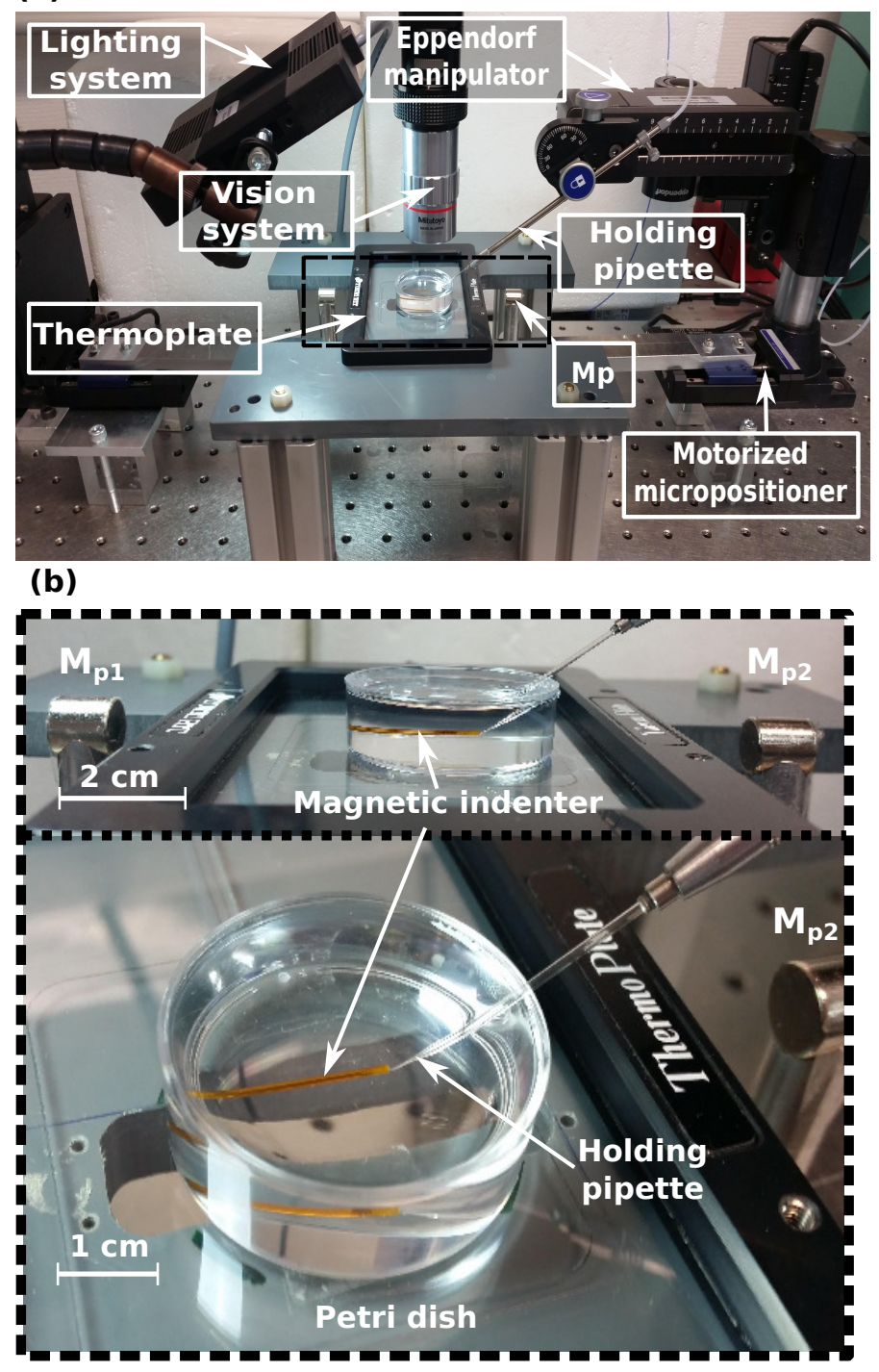

Figure 4: Experimental platform for oocytes mechanical characterisation. (a) global view of the experimental mechanical characterisation platform, (b) the loading device.

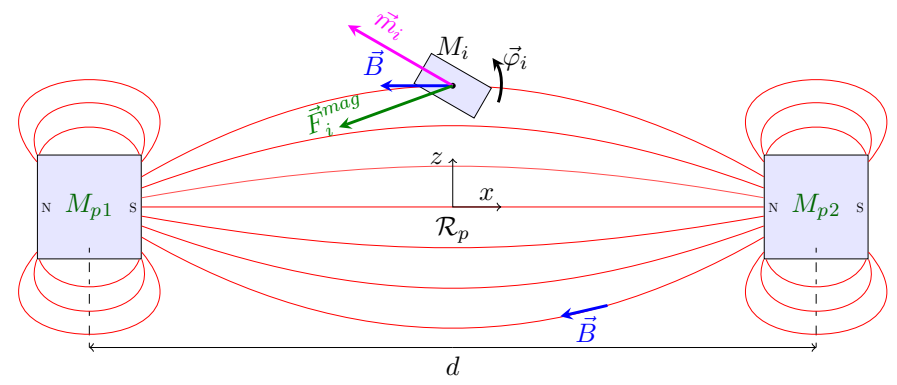

Figure 5: Modelling of the magnetic fields created by the two magnets $M_{p 1}$ and $M_{p 2}$.

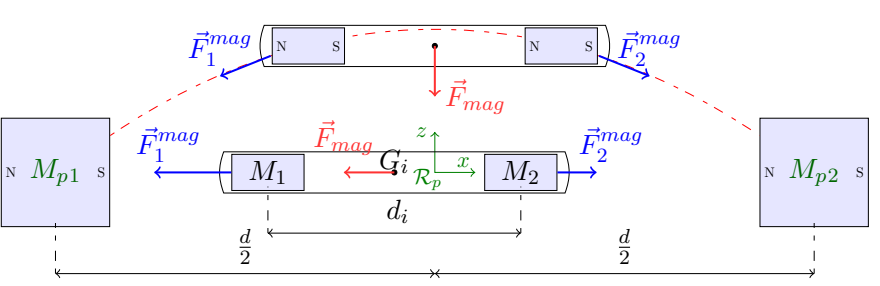

Figure 6: Modelling of the indenter and of the magnetic forces applied on it.

The entire platform is placed on an anti-vibration table to minimize the measurement noise caused by ground vibrations.

\section{Description of the force sensor}

\subsection{Magnetic modelling}

The force sensor modelling is developed in order to determine the magnetic force $\vec{F}_{m a g}$ as a function of the relative position $\vec{G}_{i}$ of the indenter from the magnets $M_{p 1}$ and $M_{p 2}$. The reference frame $R_{p}$ is defined such that its origin is placed in the middle of the segment joining $M_{p 1}$ and $M_{p 2}$, as shown in figure 5. A particular attention is paid to the $x$ component of $\vec{F}_{\text {mag }}$ because it corresponds to the normal force applied on the oocyte. Assuming that during the measurement, the acceleration and speed of the indenter are negligible, the compression force $\vec{F}_{\text {oo }}$ applied onto the oocyte remains equal to $F_{m a g}^{x}$. The determination of $F_{m a g}^{x}$ is made in a classical way. The first step consists in determining the magnetic field generated by the permanent magnets $M_{p 1}$ and $M_{p 2}$ (see figure 5 ). Here, we used an analytical expression of the magnetic field generated by a permanent magnet because it is easier to manipulate than three dimensional finite element calculation. The expression chosen here comes from Bancel [21]. It is based on the magnetic pole density $J$ to characterize the magnetization of any king of permanent magnets with two parallel pole plates (for instance parallelepipedic or cylindrical magnets). For a given magnet, its associated magnetic pole density $J$ is determined experimentally by measuring the magnetic field around the magnet with a teslameter along a direction orthogonal to the pole plates. The value of $J$ is then adjusted so that both the measured magnetic 
field and the analytical one correctly patch. Knowing $J$, allows analytically calculating the magnetic field components at any point around a single magnet $M_{p}$. The total magnetic field $\vec{B}$ is simply the sum of the magnetic field generated by $M_{p 1}$ and $M_{p 2}$ which, in our case, are placed at a constant distance $d$ from each other.

Each magnet $M_{i}(i=1,2)$ placed inside the indenter and having a magnetic dipole moment $\vec{m}_{i}$ is subject to a magnetic force (see figures 5 and 6 ):

$$
\vec{F}_{i}^{\text {mag }}=\vec{m}_{i} \cdot \nabla \vec{B}
$$

and a magnetic torque $\vec{\varphi}$ :

$$
\vec{\varphi}_{i}=\vec{m}_{i} \times \vec{B}
$$

The force $\vec{F}_{i}^{\text {mag }}$ attracts or repulses the magnet $M_{i}$ whereas the torque $\vec{\varphi}_{i}$ insures the alignment of $M_{i}$ with the magnetic field (see figure 5). The determination of $\vec{F}_{i}^{\text {mag }}$ and $\vec{\varphi}_{i}$ requires knowing the value of $m_{i}$. This $m_{i}$ value, not given by the manufacturer, is determined by means of a finite element modelling of the magnets using the COMSOL multi-physics simulation software which requires specifying $m_{i}$. This value is then adjusted such that the real magnetic flux density $\overrightarrow{B_{1}}$ generated by the magnet $M_{1}$ and measured with a teslameter is identical to the one generated by COMSOL:

$$
m_{i}=3 \cdot 10^{5} A m^{-1} .
$$

The indenter is considered as a rigid body including the two magnets $M_{i}$ on which the magnetic forces $\vec{F}_{i}^{\text {mag }}$ and torques $\vec{\varphi}_{i}$ are applied according to equations (1) and (2). $\vec{F}_{\text {mag }}$ is the resultant magnetic force applied to the indenter center of gravity $G_{i}=\left(\begin{array}{lll}G_{i}^{x} & G_{i}^{y} & G_{i}^{z}\end{array}\right)^{T}$ and is defined by:

$$
\vec{F}_{m a g}=\vec{F}_{1}^{m a g}+\vec{F}_{2}^{m a g} .
$$

\subsection{Magnetic simulation results}

A simulator was developed in order to validate the force sensor concept. This simulator includes:

- the analytical computation of the total magnetic field $\vec{B}$ generated by both magnets $M_{p 1}$ and $M_{p 2}$,

- the computation of the magnetic forces and torques applied to the indenter with the analytical model previously elaborated,

- the computation of the 6-DOF static and dynamic behavior of the indenter using Matlab/Simulink and S-functions implemented in $\mathrm{C}$ language (cf. figure 7).

Only the static behavior is investigated in this article. Due to the symmetry of the magnetic field $\vec{B}$ around the $x$ axis, the results concerning the magnetic force $\vec{F}_{\text {mag }}$ will

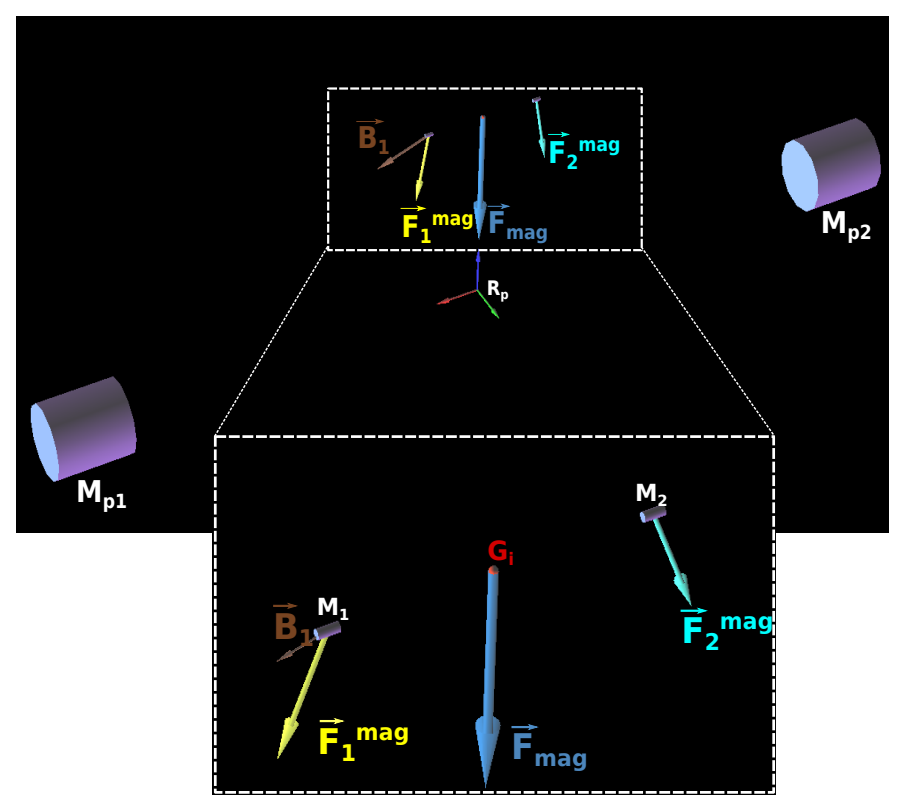

Figure 7: Simulation of the indenter behaviour.

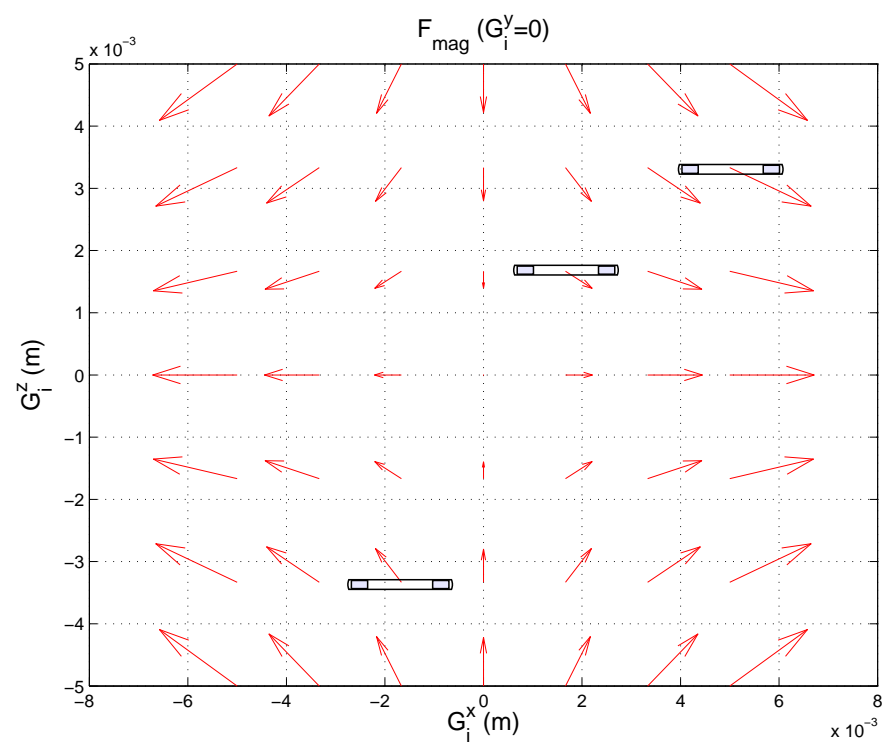

Figure 8: Mapping of the magnetic forces applied on the indenter. 


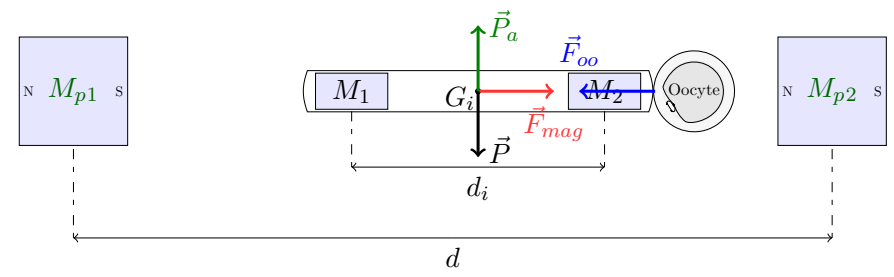

Figure 9: Equilibrium of the indenter.

be given in the plane $(x O z)$. All the simulation parameters are detailed in section 3.4. Figure 8 illustrates the evolution of $\vec{F}_{m a g}$ applied to the indenter as a function of the indenter position $G_{i}$ when the orientation of the indenter is forced to remain collinear to the $x$ axis. When the indenter is placed at $G_{i}=\left(\begin{array}{lll}0 & 0 & 0\end{array}\right)^{T}, \vec{F}_{m a g}$ is equal to zero. However, any slight perturbation or vibration will induce a slight change in the indenter position which will become $G_{i}=\left(\begin{array}{lll}\epsilon_{x} & \epsilon_{y} & \epsilon_{z}\end{array}\right)^{T}$. For example, if each $\epsilon_{i}$ is taken positive, the $x$ component of the resultant force $\vec{F}_{\text {mag }}$ becomes positive. The indenter will then leave its unstable equilibrium position in the direction of $M_{p 2}$. At the same time, the $z$ (or $y$ ) component of $\vec{F}_{\text {mag }}$ is negative, forcing the indenter to return to the $x$ axis. Therefore the specific shape of the total magnetic field force applied to the indenter implies that the $y$ and $z$ axis are the stable directions of this device while $x$ is the unstable one.

In order to obtain a stable magnetic spring, a force field $\vec{F}_{o o}$ must be added in such a way that the vector field $\vec{F}_{\text {mag }}+\vec{F}_{\text {oo }}$ becomes oriented towards an equilibrium point which does not exist in the vector field $\vec{F}_{\text {mag }}$ represented in figure 8. In other words, the $x$ component of $\vec{F}_{m a g}+\vec{F}_{o o}$ and the $x$ component of $\vec{F}_{m a g}$ must be of opposite sign. This can be obtained with a repulsion force $\vec{F}_{o o}(x)$ exhibiting an appropriate amplitude for each $x$ along this direction. The nature of this repulsion force can not be magnetic because it is proven that a static magnetic force field can not generate a stable magnetic configuration (Earnshaw's theorem). In the design proposed here, a mechanical repulsion force field along $x$ is chosen. This force is the oocyte reaction force. As shown in figure 9, when the oocyte is in contact with the indenter, it applies a reaction force $\vec{F}_{o o}$ opposite to $\vec{F}_{m a g}$ insuring its stabilization. The indenter stabilization is detailed in section 3.3.

As the indenter is placed in a culture medium which is a liquid, the up-thrust buoyancy applies a vertical ascending force $\vec{P}_{a}$ to it. The volume $v_{i}$ of the indenter is adjusted such that the module of its weight $\vec{P}$ is as close as possible to the module of $\vec{P}_{a}$. Respecting this condition, the indenter remains positioned on a vertical location which is very close to the $x$ axis located between the two magnets $M_{p i}$.

\subsection{Indenter stabilisation along $x$ axis}

The indenter of mass $m$ is, besides its weight $\vec{P}$ and the up-thrust buoyancy force $\vec{P}_{a}$ that cancel each other, under the influence of two forces: the magnetic force $\vec{F}_{\text {mag }}$ and the viscous force $\vec{F}_{v i s}$. The $x$ component of these forces can be approximated by:

$$
\begin{gathered}
F_{m a g}^{x}=K_{m a g} x \\
F_{v i s}^{x}=-K_{v} \dot{x}
\end{gathered}
$$

where $K_{m a g}$ is the magnetic spring stiffness, $K_{v}$ is the viscous coefficient and $x$ the indenter position expressed in the laboratory reference frame. The dynamic equation of the indenter is presented as:

$$
m \ddot{x}+K_{v} \dot{x}-K_{\text {mag }} x=0
$$

The equation (7) is a typical linear equation of an unstable second order system. When the oocyte is placed against the indenter, a reaction force is added. It can be approximated as follows:

$$
F_{o o}^{x}=-K_{o o} x
$$

where $K_{o o}$ is the oocyte stiffness for a given $x$. Therefore, considering this supplementary force, the dynamic equation of the indenter (7) (8) becomes:

$$
m \ddot{x}+K_{v} \dot{x}+\left(K_{o o}-K_{m a g}\right) x=0
$$

Equation (9) demonstrates that the indenter stability depends on the sign of $\left(K_{o o}-K_{m a g}\right)$. Consequently, the oocyte stiffness $K_{o o}$ must be higher or equal to the magnetic spring stiffness $K_{m a g}$ in order to insure the indenter equilibrium. Under these conditions, the force field $\vec{F}_{o o}$ prevents the indenter from moving further in the direction of $M_{p 2}$ and stabilizes it along the $x$ direction, making possible to reach a static equilibrium. When $\ddot{x}$ and $\dot{x}$ become negligible after the indenter stabilization, it turns out that:

$$
F_{o o}^{x}=K_{m a g} x
$$

\subsection{Mechanical characteristics}

The mass of the indenter is $11.32 \mathrm{mg}$. Table 1 recapitulates the characteristics of each magnet used in the real platform and in the simulation. All the magnets are characterized by both their dimensions and their gap, i.e. the distance between each magnets couples $M_{p i}$ and $M_{i}$ (see figure 9). The magnets $M_{p 1}$ are characterized by their magnetic pole density $J$ which facilitates the calculation of the external magnetic field [21]. The magnets $M_{i}$ are characterized by their magnetic dipole moment $m_{i}$ which is necessary for both magnetic force and torque calculation (Eq. (1) and (2)).

Figure 10 presents the variation of the magnetic force applied to the indenter when it moves between the two magnets $M_{p 1}$ and $M_{p 2}$ along the axis $x$, taking $G_{i}^{y}$ and $G_{i}^{z}$ 


\begin{tabular}{|l|c|c|c|c|}
\cline { 2 - 5 } \multicolumn{1}{c|}{} & $\mathrm{J}_{\left(\mathrm{A} / \mathrm{m}^{2}\right)}$ & $m_{i(\mathrm{~A} / \mathrm{m})}$ & Dimensions $(\mathrm{mm})$ & $\mathrm{Gap}_{(\mathrm{cm})}$ \\
\hline$M_{p i}$ & 1.25 & - & $\phi 10 \times 10$ & $d=10.4$ \\
\hline$M_{i}$ & - & $3.10^{5}$ & $\phi 0.5 \times 1$ & $d_{i}=1.5$ \\
\hline
\end{tabular}

Table 1: Characteristics of magnets used for the simulation.

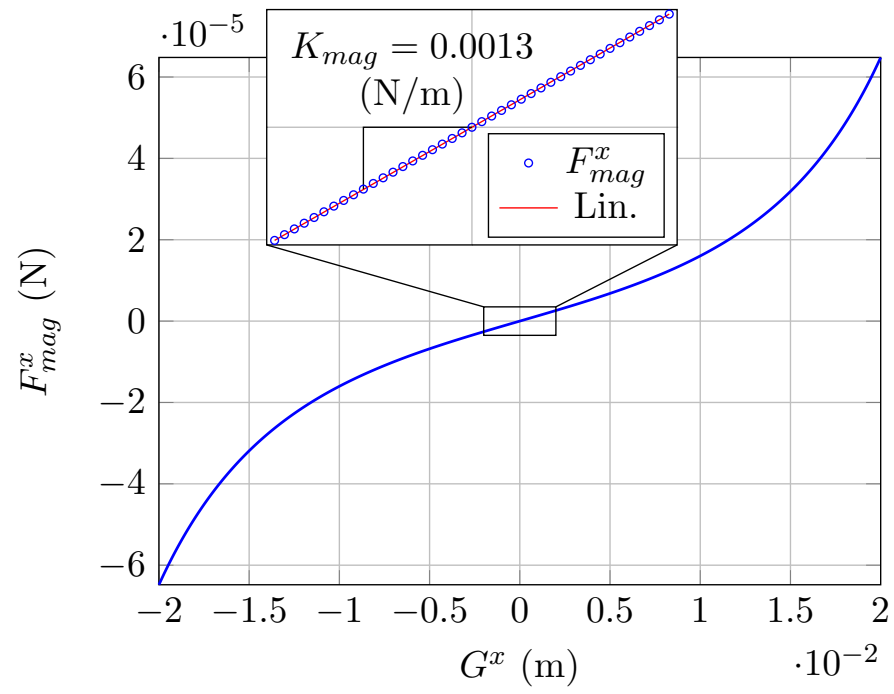

Figure 10: Magnetic force applied on the indenter when it moves along the $\mathrm{x}$ axis of the reference frame $R_{p}$.

equal to zero. This curve illustrates the instability of the indenter in the $x$ direction when no oocyte is present. In the range $-1 \mathrm{~mm}<G_{i}^{x}<1 \mathrm{~mm}$ the linearity of the curve is excellent. When an oocyte is maintained against the indenter, a usable approximation $\hat{F}_{\text {oo }}$ of the $x$ component of $\vec{F}_{o o}$ is provided as follows:

$$
\hat{F}_{o o}=K_{m a g} \cdot G_{i}^{x}=-F_{m a g}^{x} .
$$

$K_{\text {mag }}$ is the stiffness of the magnetic spring. In quasistatic conditions, the force applied to the oocyte can be easily determined knowing $K_{\text {mag }}$ and the indenter position in the interval [-1 $\mathrm{mm}, 1 \mathrm{~mm}]$ in the reference frame $R_{p}$. During the mechanical characterization of oocytes on the experimental platform, the total indenter displacement expressed in the reference frame $R_{p}$, does not exceed $300 \mu \mathrm{m}$.

Now, figure 11 shows the curve of the magnetic force applied to the indenter when it moves this time along the $z$ axis taking $G_{i}^{x}$ and $G_{i}^{y}$ equal to zero. This curve illustrates the natural stability provided by the magnetic force applied on the indenter in this direction. It also shows the intensity of the force applied to maintain the indenter along the $x$ axis. For instance, if the volume of the indenter is not correctly adjusted, the up-thrust buoyancy $P_{a}$ can be a little greater or lower than the weight $P$. Then the difference $\left|P_{a}-P\right|$ should be lower than $7 \mu \mathrm{N}$ in order to keep the indenter trapped between the magnets $M_{p i}$. A reasonable allowed distance of $2 \mathrm{~mm}$ between the indenter and $x$ horizontal axis leads to a discrepancy of $P_{a}$ equal to

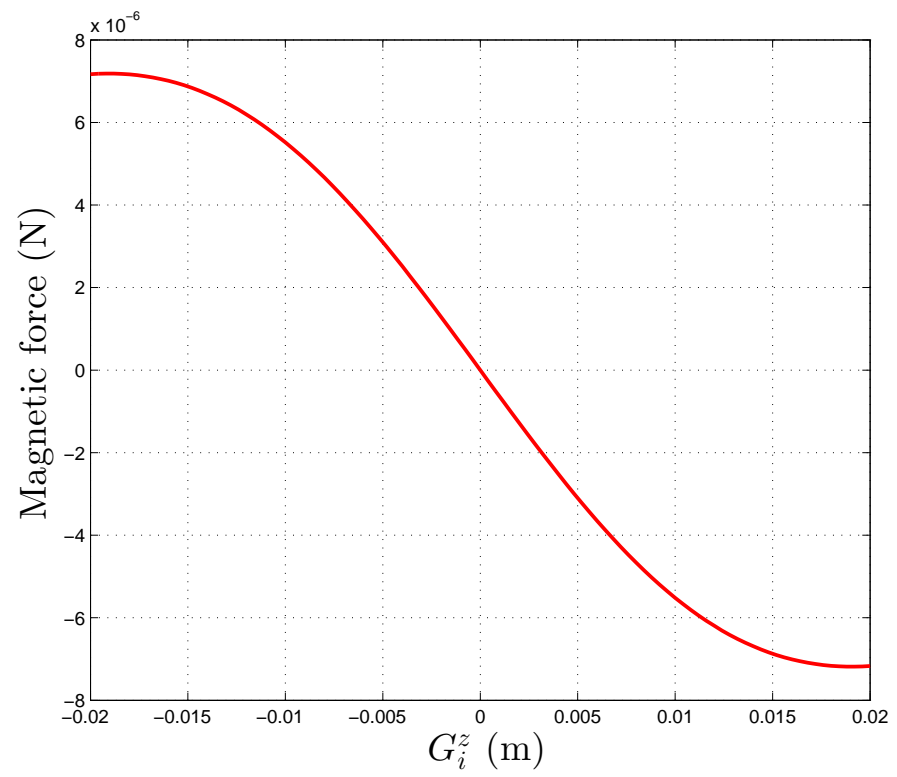

Figure 11: Magnetic force applied on the indenter when it moves along the $\mathrm{z}$ axis of the reference frame $R_{p}$.

$1.2810^{-6} \mathrm{~N}$. Consequently, the volume of the indenter has to be adjusted with a tolerance of $1.7 \mathrm{~mm}^{3}$, considering the culture medium density equal to 1 .

\section{Experimental characterisation of human oocytes}

\subsection{Oocyte preparation}

The oocytes used in this study are provided by Besançon University Hospital with the approval of its clinical ethics committee on June 9th 2010. During the ICSI protocol, the oocytes are collected and treated in order to eliminate the cumulus cells surrounding them with a hyaluronidase digestion for about 20 seconds (80 IU Hyaluronidase in Flushing, Fertipro, Belgium), a mechanical removal of the cumulus using a denudation pipettes and an IVF medium rinsing. Then they are individually observed in an inverted microscope to evaluate their maturity stage. The mature oocytes (at the metaphase II stage) are fertilised by injecting a spermatozoa into the oocyte cytoplasm, whereas the immature oocytes are incubated in the IVF medium at $37^{\circ} \mathrm{C}$ and $5 \% \mathrm{CO} 2$ for a duration of 1 to 3 hours, until the mechanical characterization is performed. The immature oocytes are exclusively intended for the study of their mechanical properties and nothing more. However, during the incubation, some of these oocytes reach the mature stage MII. Oocytes used in this study can then be at an immature stage (prophase I, metaphase I) or at a mature stage MII (immature oocytes which reach MII).

\subsection{Indenter position determination}

The vision system placed above the mechanical characterization platform not only allows observing the oocyte during the characterization but also determining the indenter position $\vec{P}_{i}$ according to the laboratory reference 
frame $R$. To do this, a normalized cross-correlation method is employed. This method evaluates the degree of similarity between two images. A picture is taken using the vision system and a distinguished sub-region of the indenter is chosen (see figure 12.a). This sub-region is the template that is sought in the main image (see figure 12.b). Once the correlation between the main image and the template is accomplished, the coordinates in pixel is recovered with the cross-correlation matrix maximum $C_{\max }=\left(\begin{array}{ll}x & y\end{array}\right)^{T}$ (see figure 12.c). The coefficient between the indenter position $\vec{P}_{i}$ given in pixels and the position given in meters is determined off-line at the beginning of the test using a calibration of the camera. The first component called $x$ of the displacement $\vec{P}_{i}$ is used to estimate $\hat{F}_{o o}$. A reference frame transformation is necessary because equation (10) is expressed in $R_{p}$ contrary to $x$ given in the fixed laboratory reference frame $R$. It comes:

$$
G_{i}^{x}=x-x_{P I}
$$

with $x_{P I}$ the displacement of the PI translation stages corresponding to the distance between the origins of $R_{p}$ and $R$.

\subsection{Experimental results}

For each oocyte characterization, the indenter is placed inside the Petri dish previously filled with IVF medium, making it floating at mid-water. The magnets positions are initialized using the PI translation stages in such a way that the indenter stays in contact against the Petri dish border. The oocyte is picked up with a Pasteur pipette and released into the Petri dish. The oocyte is immobilized and placed at a few micrometers away from the tip of the indenter by means of a holding pipette controlled by an Eppendorf manipulator. Then, the operator actuates simultaneously both PI stages using a synchronous command in order to bring the magnet $M_{P 2}$ closer to the indenter (see figure 9 ). By the action of the magnetic force $\vec{F}_{m a g}$, the indenter moves in the direction of $M_{P 2}$ and leans on the oocyte external zona pellicida without deforming it. The purpose of this operation is to change its equilibrium point from the Petri dish border to the oocyte side. For the determination of the indenter position as mentioned in section 4.2, a sub-region of the indenter (template) is selected in the image provided by the microscope and the vision system.

Figure 13 shows the oocyte mechanical response to a loading/unloading cycle consisting in compressing the oocyte with the indenter until the compression length of the oocyte $d_{o o}$ reaches $14 \mu \mathrm{m}$ followed by an unloading until the indenter returns to its initial position. The cycle is driven automatically by a script. Between two measurements in quasi-static conditions, the indenter displacement is performed by simultaneously moving the magnets $M_{p 1}$ and $M_{p 2}$ with a synchronous command of the two PI stages while the holding pipette stays fixed during the entire test. a)

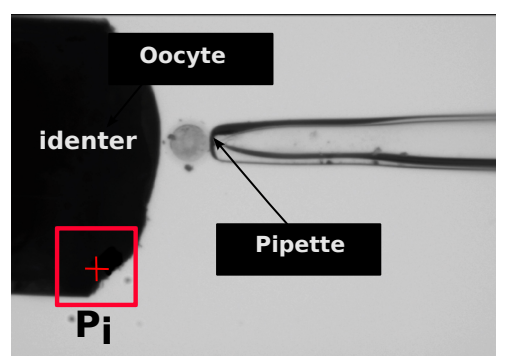

b)

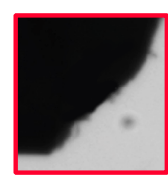

c)

i.

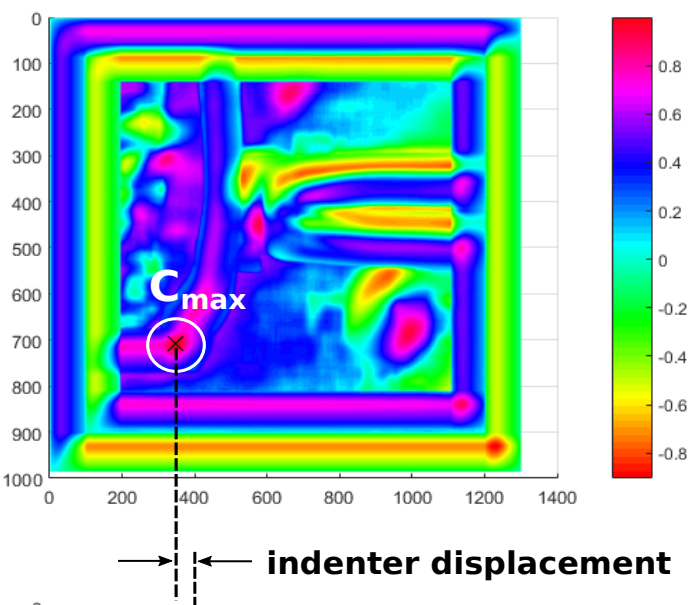

ii.

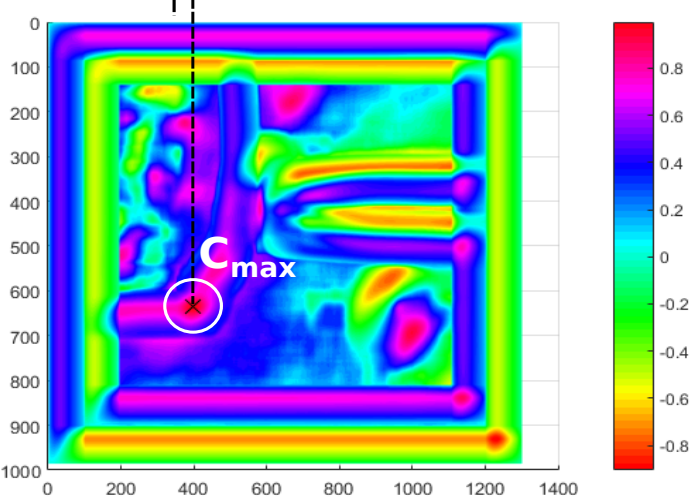

Figure 12: Normalized cross-correlation method, a) sub-region selection, b) pattern, c) representation of the cross-correlation matrix and determination of $C_{\max }(\mathrm{i})$ before the indenter displacement and (ii) after the indenter displacement. 


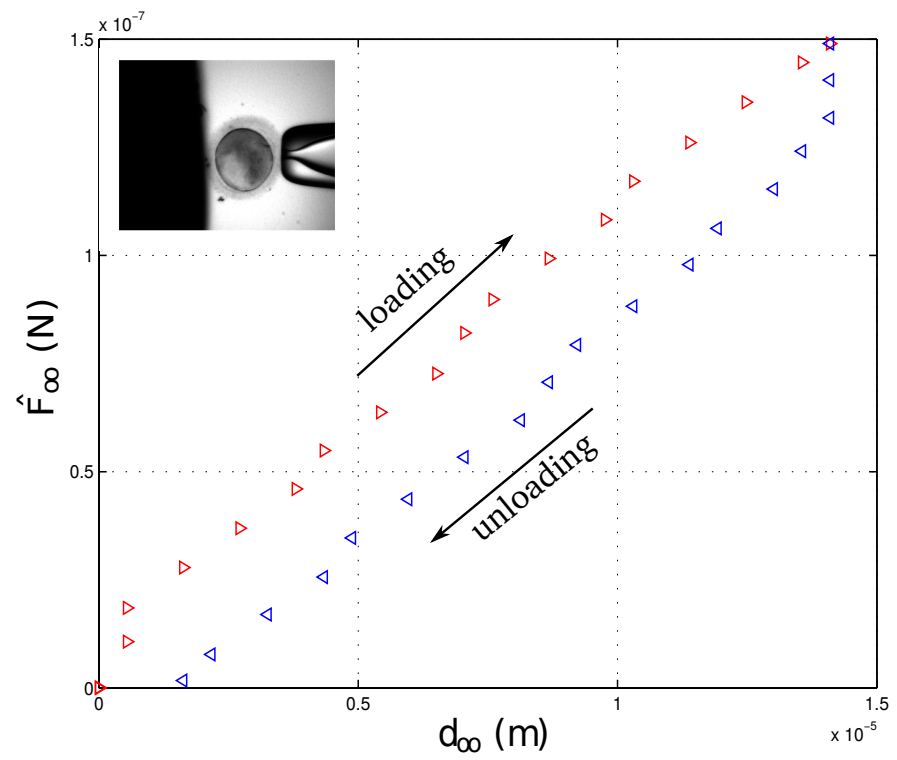

Figure 13: Oocyte $O_{1}$ mechanical behavior

The script insures a constant gap $d$ between the external magnets. The PI stages speed is equal to $6 \mu \mathrm{m} / \mathrm{s}$. The oocyte compression length $d_{o o}$ is calculated from the difference between the initial indenter position and its position $x$ during the cycle. At the beginning of the characterization, $d_{o o}$ is equal to zero when the indenter is in contact with the oocyte without deforming it. The determination of $\hat{F}_{o o}$ is done using equations (10) and (11):

$$
\hat{F}_{o o}=K_{\text {mag }}\left(x-x_{P I}\right)
$$

The determination of an accurate value of the magnetic spring stiffness $K_{m a g}$ is quite difficult and remains an important challenge. We decided to initially work with an approximation of this value given by the model described in figure 10 and where all the simulation parameters have been identified in accordance with the experiments. So $K_{\text {mag }}$ is supposed to be equal to $0.0013 \mathrm{~N} / \mathrm{m}$. The order of magnitude of this value has been validated using an identification method described in the next section. This stiffness is very low compared to the lowest stiffness of Atomic Force Microscope cantilevers which are commercially available (around $0.01 \mathrm{~N} / \mathrm{m}$ ). The very low stiffness of this device is achieved due to the large distance $d$ between the external magnets.

The curve shown in figure 13 presents the results obtained with an immature oocyte (metaphase I) labelled $O_{1}$. Oocyte $O_{1}$ exhibits a diameter $\phi_{p}$ equal to $144 \mu \mathrm{m}$ and a cytoplasm diameter $\phi_{c}$ equal to $106 \mu \mathrm{m}$. The oocyte behaviour during the loading cycle is linear. The difference detected between the load and the unload is the hysteresis due to the viscoelastic nature of the oocyte in accordance with the results obtained by Abadie et al. [17].

\section{Calibration of the nano-force sensor}

Calibrating a nanoforce sensor is still an important challenge that the National Metrology Institutes (NMIs) try to develop [? ]. Knowing that there is neither force standards or normalization for small forces below the millinewton, we decided to develop our own procedure. The approach consists in measuring the displacement $s x$ of the indenteur that corresponds to the transient response of the equation (7) and where $F_{o o}$ is equal to zero. The determination of the indenter mass can be easily performed using a precision balance. Having experimental values of $x$ in this conditions, theoretically permit to determine the two unknown parameters $K_{v}$ and $K_{\text {mag }}$ of the zero input equation (7). This free response is obtained by measuring the indenter displacement $x$ when it is free to move, i.e when it is not in contact either against the Petri dish or the oocyte. This measurements has been realized using the image processing algorithm for the determination of the indenter position detailed in section 4.2. The procedure for initiating the indenter free displacement is the following:

- initially the indenter is in contact against the Petri dish border,

- the external magnets are simultaneously moved in order to cancel then inverse the magnetic force $F_{m a g}^{x}$ which maintains the indenter against the Petri dish,

- once $F_{m a g}^{x}$ is greater then the adhesion force between the Petri dish and the indenter, this one moves under the influence of the force $F_{m a g}^{x}$ towered the pipette placed at a distance that assure a displacement of $180 \mu \mathrm{m}$,

- during the zero input movement of the indenter, an sequence acquisition is performed at a frame rate of $40 \mathrm{images} / \mathrm{s}$ (the maximum value for our camera),

- all the images acquired during the sequence are individually post processed in order to extract $x$ and the corresponding time $t$ provided by the internal trigger of the camera.

The experimental measurement of this zero input response is given in figure 14 . The parameters $K_{v}$ and $K_{m a g}$ are identified using a least squares optimization algorithm that best approximates the simulated and the experimental free response. Equation (7) has a solution of the following form:

$$
x(t)=\lambda_{1} e^{a_{1} t}+\lambda_{2} e^{a_{2} t}
$$

with constants $\lambda_{1}, \lambda_{2}, a_{1}$ and $a_{2}$ which depends on $m, K_{v}$, $K_{\text {mag }}$, initial conditions $x(0)$ and $\dot{x}(0)$. Using Matlab, we superpose on figure 14 the experimental free response and the one obtained by the model. The two parameter's values obtained by this calibration test are:

$$
\left\{\begin{aligned}
K_{v} & =8.96 \cdot 10^{-5} \mathrm{~N} . \mathrm{s} / \mathrm{m} \\
K_{\text {mag }} & =0.00147 \mathrm{~N} / \mathrm{m} .
\end{aligned}\right.
$$




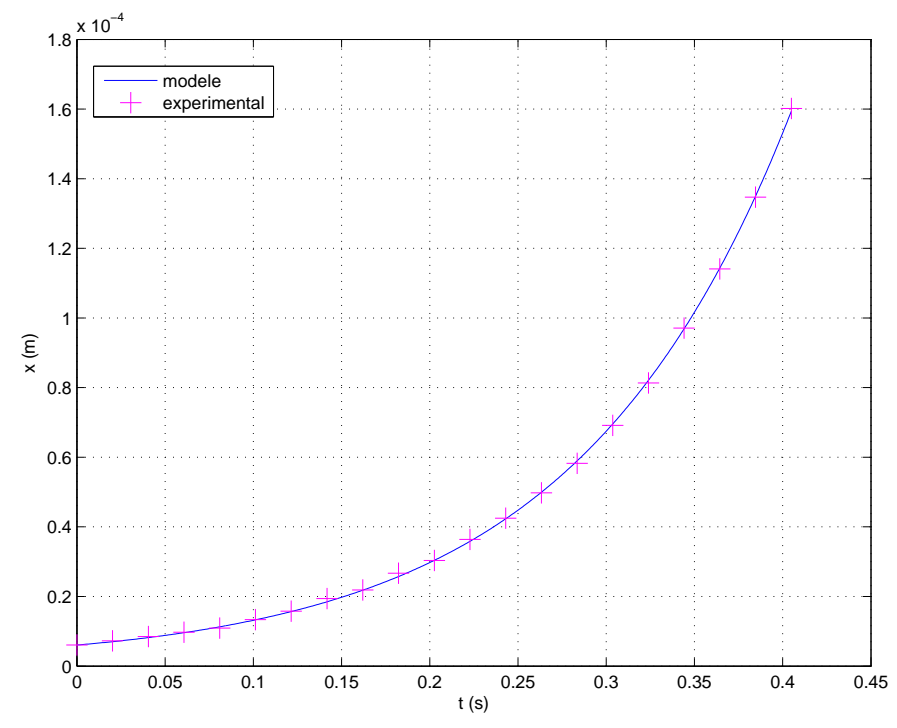

Figure 14: Indenter experimental zero input response axis and comparison with the dynamic model for the nano-force sensor calibration.

The order of magnitude previously found by simulation $\left(K_{m a g}=0.0013 \mathrm{~N} / \mathrm{m}\right)$ is confirmed by this calibration test.

\section{Conclusion and Perspectives}

A new platform, specially designed for a low cost mechanical characterization of human oocytes, is proposed. It is composed of a micro and nano force sensor based on passive magnetic springs, a vision system and a Petri dish in which the mechanical characterization of the oocyte is performed. This force sensing platform meets all the conditions imposed by the French law concerning bioethics. Indeed, the transducer of the force sensor is a glass capillary containing two magnets. The oocyte is placed inside a common Petri dish used in ICSI routine protocol. The magnetic modelling of the force sensor was realized. The magnetic force $F_{m a g}^{x}$ applied on the oocyte by the magnetic indenter along the $x$ axis is linear with respect to the indenter displacement $G_{i}^{x}$ and, in quasi-static conditions, equal to the oocyte reaction $F_{o o}$. Therefore knowing the magnetic stiffness of the force sensor $K_{\text {mag }}$ and the indenter displacement $G_{i}^{x}$, an estimation $\hat{F}_{\text {oo }}$ of the $x$ component of $\vec{F}_{\text {oo }}$ can be calculated. Preliminary results obtained with an experimental platform were presented. These results showed that the oocyte behaviour is linear during a loading cycle with a compression length which is not exceeding $15 \mu \mathrm{m}$. Unlike the majority of force sensors which are stable, the measurement performed with the force sensor proposed in this article is done along an unstable direction which is passively stabilized by the oocyte mechanical reaction. This is an original approach for force sensors based on passive magnetic spring. In order to determine with accuracy the sensor magnetic stiffness $K_{\text {mag }}$, a calibration approach has been developed. The calibration test has revealed that the order of magnitude of $K_{\text {mag }}$ is $0.00147 \mathrm{~N} / \mathrm{m}$, which is a very low stiffness compared to the stiffness of other force sensors like AFM. Further study will have to be conducted to validate, by performing several series of calibrations, not only the value but also the uncertainty range of the magnetic stiffness $K_{m a g}$.

\section{Acknowledgments}

This work has been supported by the MICROBE Regional project of Franche-Comté (contract "2013C-5497") and by the Labex ACTION project (contract "ANR-11LABX-0001-0").

[1] R. P. MARRS, "Human in-vitro fertilization.," Clinical obstetrics and gynecology, vol. 29, no. 1, p. 117, 1986.

[2] A. Van Steirteghem, Z. Nagy, J. Liu, H. Joris, G. Verheyen, J. Smitz, H. Tournaye, I. Liebaers, and P. Devroey, "Intracytoplasmic sperm injection," Baillière's clinical obstetrics and gynaecology, vol. 8, no. 1, pp. 85-93, 1994.

[3] Y.-C. Lin, S.-Y. Chang, K.-C. Lan, H.-W. Huang, C.-Y. Chang, M.-Y. Tsai, F.-T. Kung, and F.-J. Huang, "Human oocyte maturity in vivo determines the outcome of blastocyst development in vitro," Journal of assisted reproduction and genetics, vol. 20, no. 12 , pp. 506-512, 2003

[4] P. Xia, "Intracytoplasmic sperm injection: correlation of oocyte grade based on polar body, perivitelline space and cytoplasmic inclusions with fertilization rate and embryo quality.," Human Reproduction, vol. 12, no. 8, pp. 1750-1755, 1997.

[5] Q. Wang and Q.-Y. Sun, "Evaluation of oocyte quality: morphological, cellular and molecular predictors," Reproduction, Fertility and Development, vol. 19, no. 1, pp. 1-12, 2006.

[6] C. Pieralli, B. Wacogne, C. André, C. Roux, C. Joanne, and L. Pazart, "Biological qualification of oocyte maturity with the use of the karhunen-loeve transform: Computer-aided decision for selecting best oocytes before fertilization," in 1st International Workshop on Medical Image Analysis and Description for Diagnosis Systems, pp. 77-84, 2009.

[7] A. Beuchat, P. Thévenaz, M. Unser, T. Ebner, A. Senn, F. Urner, M. Germond, and C. Sorzano, "Quantitative morphometrical characterization of human pronuclear zygotes," Human Reproduction, vol. 23, no. 9, pp. 1983-1992, 2008.

[8] C. Pelletier, D. L. Keefe, and J. R. Trimarchi, "Noninvasive polarized light microscopy quantitatively distinguishes the multilaminar structure of the zona pellucida of living human eggs and embryos," Fertility and sterility, vol. 81, pp. 850-856, 2004.

[9] F. Vidberg, R. Zeggari, C. Pieralli, C. Amiot, C. Roux, and B. Wacogne, "Measurement of oocyte temporal maturation process by means of a simple optical micro-system," Sensors and Actuators B: Chemical, vol. 157, no. 1, pp. 19-25, 2011.

[10] R. Zeggari, B. Wacogne, C. Pieralli, C. Roux, and T. Gharbi, "A full micro-fluidic system for single oocyte manipulation including an optical sensor for cell maturity estimation and fertilisation indication," Sensors and Actuators B: Chemical, vol. 125, no. 2, pp. 664-671, 2007.

[11] L. Z. Yanez, J. Han, B. B. Behr, R. A. R. Pera, and D. B. Camarillo, "Human oocyte developmental potential is predicted by mechanical properties within hours after fertilization," Nature communications, vol. 7, 2016.

[12] B. Wacogne, C. Pieralli, C. Roux, and T. Gharbi, "Measuring the mechanical behaviour of human oocytes with a very simple su-8 micro-tool," Biomedical Microdevices, vol. 10, no. 3, pp. 411-419, 2008.

[13] X. Liu, R. Fernandes, A. Jurisicova, R. F. Casper, and Y. Sun, "In situ mechanical characterization of mouse oocytes using a cell holding device," Lab on a Chip, vol. 10, no. 16, pp. 21542161, 2010.

[14] Y. Sun, K.-T. Wan, K. P. Roberts, J. C. Bischof, and B. J. Nelson, "Mechanical property characterization of mouse zona pel- 
lucida," NanoBioscience, IEEE Transactions on, vol. 2, no. 4, pp. 279-286, 2003.

[15] Y. Murayama, C. E. Constantinou, and S. Omata, "Micromechanical sensing platform for the characterization of the elastic properties of the ovum via uniaxial measurement," Journal of biomechanics, vol. 37, no. 1, pp. 67-72, 2004.

[16] S. Sakuma and F. Arai, "Cellular force measurement using a nanometric-probe-integrated microfluidic chip with a displacement reduction mechanism," J. Robot. Mechatron, vol. 25, no. 2, pp. 277-284, 2013.

[17] J. Abadie, C. Roux, E. Piat, C. Filiatre, and C. Amiot, "Experimental measurement of human oocyte mechanical properties on a micro and nanoforce sensing platform based on magnetic springs," Sensors and Actuators B: Chemical, vol. 190, pp. 429438, 2014.

[18] A. Cherry, J. Abadie, and E. Piat, "Analysis of a passive microforce sensor based on magnetic springs and upthrust buoyancy," Sensors and Actuators A: Physical, vol. 169, no. 1, pp. 27-36, 2011.

[19] S. Sakuma, B. Turan, and F. Arai, "High throughput mechanical characterization of oocyte using robot integrated microfluidic chip," in Intelligent Robots and Systems (IROS), 2013 IEEE/RSJ International Conference on, pp. 2047-2052, IEEE, 2013.

[20] J. Abadie, E. Piat, S. Oster, and M. Boukallel, "Modeling and experimentation of a passive low frequency nanoforce sensor based on diamagnetic levitation," Sensors and Actuators A: Physical, vol. 173, no. 1, pp. 227-237, 2012.

[21] F. Bancel and G. Lemarquand, "Three-dimensional analytical optimization of permanent magnets alternated structure," $\mathrm{Mag}$ netics, IEEE Transactions on, vol. 34, pp. 242-247, Jan 1998. 